\title{
OEPA-COPP Regimen
}

National Cancer Institute

\section{Source}

National Cancer Institute. OEPA-COPP Regimen. NCI Thesaurus. Code C67170.

A regimen containing vincristine, etoposide, procarbazine and doxorubicin (OEPA)

followed by cyclophosphamide, vincristine, prednisone and procarbazine (COPP), given in

combination with radiation therapy for the treatment of male patients with intermediatehigh risk, childhood Hodgkin lymphoma. 